\title{
MEATBORNE PARASITIC ZOONOSIS
}

\author{
Uğur Uslu' and Bayram Șenlik ${ }^{2}$
}

'Selcuk University, Faculty of Medicine, Department Microbiology, 42250 Konya, Turkey.

${ }^{2}$ Uludağ University, Faculty of Veterinary Medicine, Department of Parasitology, Gorukle Campus, 16059, Bursa, Turkey.

*Corresponding author: uuslu69@gmail.com

\section{INTRODUCTION}

Foodborne parasites seriously threaten human health in many parts of the world. Besides affecting human health, parasites adversely affect the export of animal products from countries with a disease to other countries. Parasitic diseases affect meat and milk production, and reproductive performance of the animals they infect, and thus, cause loss of millions of dollars by reducing the quality of the skin and fleece. Therefore, foodborne zoonoses can serve as a commercial barrier among countries. The prevalence of foodborne parasites can vary among countries and even between different regions of the same country. Factors contributing to this variation include differences in animal breeding systems, demographic structure, and nutritional habits (Macpherson et al. 2000; Slifko et al. 2000; Gupta et al. 2018; Zarlenga and Gamble 2019).

Parasitic diseases continue to be an important public health problem in both developed and developing countries. Data from the World Health Organization (WHO) estimate that one of every four people worldwide is infected with parasites (WHO 2021). Due to the rapid increase in the world population, more protein sources are needed every day. In addition, as seen during the COVID-19 epidemic, the food supply chain becomes of paramount importance in pandemics that affect the whole world at the same time. Therefore, it is clear that food safety will continue to be an important issue in the future.

Foods of animal origin play an important role in providing people with balanced and adequate nutrition (Dhaliwal and Juyal 2013). According to the Food and Agriculture Organization of the United Nations (FAO), at least $40-50 \%$ of daily protein needs of humans should be obtained from foods of animal origin for a balanced diet. The FAO has recommended that 44 grams of the daily protein need out of 90 grams should be of animal origin. However, the worldwide daily consumption of animal protein per person is approximately 32 grams. Based on this data, it seems that many people do not consume enough animal protein.

Meat is the most accessible animal protein source. People meet protein needs by eating the meat of various animals, including game animals. However, the meat can cause viral and bacterial diseases, as well as directly or indirectly transmit various parasitic diseases, to humans if it are not subjected to a proper and meticulous examination.
Compared to other foodborne parasitic diseases, the number of those transmitted by meat is small, but their consequences in humans can be much more severe (Zhou et al. 2008; Bhatia et al. 2010; Dhaliwal and Juyal 2013). For instance, various growth stages of the Toxoplasma gondii, which are found in the muscles and edible internal organs of infected animals, are transmitted to humans through meat that is eaten raw or undercooked. The prevalence of diseases with meat borne zoonoses is higher in underdeveloped or developing countries, where meat is generally consumed unprocessed, than in developed countries (Gupta et al. 2018). In such countries, animals are usually slaughtered outside slaughterhouses, and the meat of animals slaughtered in slaughterhouses is typically not adequately inspected by veterinarians. Therefore, meat carrying the infective forms of parasites quickly enters public circulation and is consumed. In addition, people's lack of knowledge about parasitic diseases transmitted from meat accelerates this spread.

In recent years, various tests with high specificity and sensitivity have been developed to facilitate the diagnosis of parasitic diseases in humans and animals. In addition, important developments have been made in the effective treatment of people, who contract these diseases. Further, strict legal regulations regarding meat inspection have been implemented, especially in European countries. Despite these developments, various precautions have not completely prevented the transmission of parasitic diseases by meat. Accordingly, more effective measures must be taken for safer meat consumption and to raise people's awareness about these diseases and their means of transmission.

In the next section, parasitic zoonoses transmitted directly to humans through meat will be outlined. However, diseases such as Echinococcosis, which are transmitted to definitive hosts through meat or other means and then infect humans, will not be discussed.

Parasitic diseases transmitted to humans by consuming the meat of various animals are Taeniasis, Trichinellosis, Toxoplasmosis, and Sarcocystosis (Gamble 1998; Ünüvar 2018; Liu 2019; Zarlenga and Gamble 2019). The parasitic factors that cause these diseases are outlined in Table 1.

\section{TAENIASIS}

Three main types of Taenia infect humans; these include Taenia solium, Taenia saginata, and Taenia asiatica. Although T. asiatica was initially considered a strain or 
subspecies of $T$. saginata, subsequent morphological and genetic studies have revealed that it is a different species. While adult forms of these species live in the small intestines of humans, metacestode forms are seen in muscles or internal organs of animals that are intermediate hosts (Ünüvar 2018; Liu 2019; Zarlenga and Gamble 2019). The larva of $T$. saginata is called Cysticercus bovis and is located in the tongue, diaphragm, heart, and striated muscles of cattle, which serve as intermediate hosts. Cysticercus cellulosae, the larva of T. solium, is mainly seen in the muscles of pigs. However, humans can be both the definitive host and intermediate host for $T$. solium, and these larvae can develop in the muscles, under the skin, or in the brain. Cysticercus viscerotropica, the larva of T. asiatica, mostly develops in the liver of domestic and wild pigs, but can sometimes be found in the lungs ( $\mathrm{Li}$ et al. 2007; Liu 2019; Zarlenga and Gamble 2019). Some of the main characteristics of the Taenia species that infect humans are summarized in Table 2 (Liu 2019).

Table 1: Main meatborne parasitic zoonoses

\begin{tabular}{lllll}
\hline Parasites group & Genus & Species & Disease & References \\
\hline Helminth & Taenia & Taenia saginata & Taeniosis & $\bullet$ Dubey (1986; 2015) \\
& & Taenia solium & Taeniosis/Cysticercosis & $\bullet$ Bhatia et al. (2010) \\
& & Taenia asiatica & Taeniosis & $\bullet$ Dhaliwal and Juyal (2013) \\
& Trichinella & Trichinella spiralis & Trichinellosis & $\bullet$ Ünüvar (2018) \\
& & and any other species & & Ortega (2019) \\
Protozoon & Toxoplasma & Toxoplasma gondii & Toxoplasmosis & $\bullet$ Liu (2019) \\
& Sarcocystis & Sarcocystis suihomonis & Sarcocystosis & Zarlenga and Gamble (2019) \\
& & Sarcocystis hominis & & \\
\end{tabular}

Table 2: Some characteristics of Taenia species that infect humans.

\begin{tabular}{|c|c|c|c|c|c|}
\hline Species & $\begin{array}{l}\text { Definitive } \\
\text { host }\end{array}$ & $\begin{array}{l}\text { Intermediate } \\
\text { host }\end{array}$ & Adult cestode & Metacestode & Prevalence \\
\hline $\begin{array}{l}\text { Taenia } \\
\text { solium }\end{array}$ & Man & $\begin{array}{l}\text { Domestic and } \\
\text { wild pig }\end{array}$ & $\begin{array}{l}\text { - Its length is between } 2-8 \mathrm{~m} \text {. } \\
\text { - There are } 1000 \text { proglottids } \\
\text { in its strobila. }\end{array}$ & 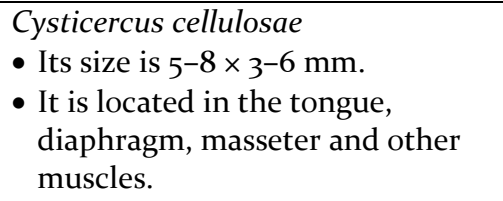 & $\begin{array}{l}\text { Cosmopolitan. } \\
\text { Common in Latin } \\
\text { America, Africa, Mexica, } \\
\text { Russia, India, Asia. }\end{array}$ \\
\hline \multirow[t]{2}{*}{$\begin{array}{l}\text { Taenia } \\
\text { saginata }\end{array}$} & Man & & $\begin{array}{l}\text { - Its length is between } \\
\text { 4-12 m. } \\
\text { - There are 10oo-20oo } \\
\text { proglottids in its strobila. }\end{array}$ & $\begin{array}{l}\text { Cysticercus bovis } \\
\text { - It is size is } 6-10 \times 4-6 \mathrm{~mm} \text {. } \\
\text { - It is located in the tongue, } \\
\text { diaphragm, masseter and other } \\
\text { muscles. }\end{array}$ & $\begin{array}{l}\text { Cosmopolitan. } \\
\text { Common in Africa, Asia, } \\
\text { Europe and America. }\end{array}$ \\
\hline & & Cattle & & $\begin{array}{l}\text { - Sometimes it can be found in the } \\
\text { liver and lungs. }\end{array}$ & \\
\hline $\begin{array}{l}\text { Taenia } \\
\text { asiatica }\end{array}$ & Man & $\begin{array}{l}\text { - Domestic and } \\
\text { wild pig } \\
\text { - Rarely cattle } \\
\text { and goats }\end{array}$ & $\begin{array}{l}\text { - Its length is } 3.5 \mathrm{~m} \text {. } \\
\text { - There are } 700-900 \\
\text { proglottids in its strobila. }\end{array}$ & $\begin{array}{l}\text { Cysticercus viscerotropica } \\
\text { - It is size is } 2 \times 2 \mathrm{~mm} \text {. } \\
\text { - It is located in the liver and lungs. }\end{array}$ & $\begin{array}{l}\text { Its prevalence is more } \\
\text { limited. } \\
\text { It is seen in countries such } \\
\text { as Taiwan, South Korea, } \\
\text { Indonesia, Japan, etc. }\end{array}$ \\
\hline
\end{tabular}

\section{T. solium TAENIASIS}

This species, known as "Pig tapeworm," is an important public health problem, causing Neurocysticercosis in people living in areas where pig breeding is common. Around 50 million people worldwide are estimated to be affected by this disease. Death occurs in $1 \%$ of the individuals affected by the disease, and reports indicate that approximately 50,000 people worldwide die from this disease every year. Therefore, WHO describes Neurocysticercosis as an important neglected tropical disease (Schantz et al. 1993; Zarlenga and Gamble 2019).

\section{Epidemiology}

T. solium, which is a worldwide common helmintozoonosis, poses a serious public health problem, especially in underdeveloped and developing countries
(Tsang and Wilson 1995; Bern et al. 1999; Murrell 2005; Juyal et al. 2008; Yanagida et al. 2012). T. solium is more prevalent in areas where pig farming is widespread, people are in close contact with pigs, and pork is consumed raw or undercooked. Humans can be intermediate hosts for this parasite, which is of great importance in epidemiology. People can consume $T$. solium eggs via contaminated food or water, or autoinfections can occur.

In such cases, the eggs may travel to the brain, causing Neurocysticercosis, or they can settle in other tissues and organs, such as muscle tissue and subcutaneously (Bruschi and Gomez-Morales 2017; Ünüvar 2018; Liu 2019; Zarlenga and Gamble 2019). Neurocysticercosis is one of the most frequent preventable causes of epilepsy in developing countries and is a long-term infection that adversely affects patients' quality of life (Flisser et al. 2005). 
T. solium infections can be seen in many countries, and are more common in Latin America, Mexico, India, Africa, non-Muslim Asian countries, China, Nepal, Russia, Southern and Eastern European countries, and Slavic countries (Geerts 1993; Schantz et al. 1998; Liu 2019; Zarlenga and Gamble 2019). Distinguishing T. solium eggs from other Taenia species in coprological examinations is very difficult. For this reason, determining the prevalence in humans precisely and accurately is not possible. Increasing illegal human migration in recent years has caused infection to spread rapidly.

\section{Morphology}

Adult forms of $T$. solium live in the small intestine of humans. Due to the presence of hooks in the rostellum, the parasite is called "armed tapeworm." Its length varies between 2-8 $\mathrm{m}$. There are approximately 1000 proglottids in its strobilas, and 22-32 hooks in two rows in its scolex. Each gravid proglottid contains approximately 40,000 eggs. T. Solium is a long-lived parasite and can survive in the human intestine for more than 10 years. $C$. cellulosae are about 3×5 mm and filled with a cloudy liquid. Eggs are spherical or slightly oval, brownish, and $40-48 \mathrm{~m} \mu$ in size (Sayg1 1999; Liu 2019).

\section{Transmission}

Humans are infected by eating the raw or undercooked cysticerci-containing meat of intermediate host (pigs). Infection can also occur by ingesting external eggs, and $C$. cellulosae can develop. Intermediate host pigs are infected by consuming food and water contaminated with eggs.

\section{Life Cycle}

The only known final host of $T$. solium is humans. Pigs normally serve as intermediate hosts. However, sometimes in pig-breeding regions, the metacestode form can be found in dogs. Eggs leave the host when proglottids break off from the parasite and are excreted in human feces. The breakdown of the proglottids in the external environment releases and spreads the eggs. When pigs consume the eggs, they open in the small intestine, releasing oncospheres. The oncospheres enter the mesenteric veins in the intestinal wall and move through the bloodstream, eventually settling in muscles or internal organs. Then, they develop into cysticerci, the larval shape, in about two months. In the early period, a fibrous capsule develops around the $C$. cellulosae as a result of the host's response. Under normal conditions, the cysticercus, which is $3 \times 5 \mathrm{~mm}$ in size, can reach a size that can contain $60 \mathrm{ml}$ of liquid in its brain and subdural areas (Sayg1 1999; Liu 2019; Zarlenga and Gamble 2019). The most common muscles, where $C$. cellulosae are found in pigs, are the heart, masseter, diaphragm, and tongue muscles. Due to their appearance in the pork meat, cysticerci are also called "pork measles" (Sayg1 1999).
People often become infected by eating raw or undercooked pork meat that carries $C$. cellulosae. Larvae separate from muscles or other tissues in the stomach, adhere to the wall of small intestine and evaginate in the upper parts of the small intestine; adult parasites develop in 5-12 weeks (Sayg1 1999; Liu 2019; Zarlenga and Gamble 2019). Humans can also be an intermediate host for this parasite. Infections in this way occurs by consuming foods contaminated with $T$. solium eggs or by transmitting eggs from the hand to the mouth. Eggs that open in the small intestines of humans, enter the blood vessels as they do in pigs, and lodge under the skin or in the muscles, eyes, tongue, and most importantly, the brain. Another form of transmission in humans is autoinfection. In humans, who carry this parasite in their small intestines, the eggs can enter the stomach through reverse peristalsis. After the oncospheres are released, they become cysticerci by traveling to various organs and tissues. Cysticerci that settle in the brain or on its surface cause neurocysticercosis (Sayg1 1999; Liu 2019; Zarlenga and Gamble 2019).

\section{Clinical Signs}

Although infected people usually have only one parasite, occasionally more parasites are found, and in some cases more than 25 parasites have been found (Sayg1 1999; Zarlenga and Gamble 2019). The hooks in the scolexes of T. Solium cause damage to the intestinal tissue where they attach, and consequently cause inflammation and pain. Intestines typically are not obstructed. However, intestinal perforation, peritonitis, and gall bladder inflammation may occur (Zarlenga and Gamble 2019). The clinical signs of adult $T$. solium infections are similar to those ofT. saginata infections. When a single parasite is found, typically no significant symptoms occur. Infection may not be noticeable, until a proglottid is seen being ejected from the anus of an individual carrying the parasite. The most common clinical symptoms are colic, abdominal pain, nausea, vomiting, and chronic dyspepsia. Abdominal pain and nausea may be seen in children, especially in the morning, and improve when a small amount of food is eaten. In addition to these symptoms, anal itching, diarrhea, constipation, weakness, anorexia, anemia, and rarely fever may be observed in infected people (Sayg1 1999; Liu 2019). In addition, blood eosinophil counts can increase by up to $28 \%$.

The main pathogenic effects in humans are caused by the larval stages, which can settle in various tissues and organs. Mild infections, in which cysticerci are not found in vital areas and the larvae are not yet developed, typically display no remarkable symptoms in humans. Cysticerci can cause neurocysticercosis, ophthalmic cysticercosis, subcutaneous cysticercosis, and muscular or lingual cysticercosis, depending on where they are located (Cardenas et al. 1992; Garcia et al. 2003; Elias et al. 2005). Clinical symptoms can also vary according to the organ infected and number of cysticerci. The most severe infection and symptoms occur in the central nervous system (CNS). The most common symptom of cysticerci 
located in the brain is epileptic seizures, which start when the larvae first settle. The death of these larvae is increased by cysting and tearing. Another common symptom is hydrocephalus, caused by blockage of the cerebrospinal fluid (CSF). In addition, mental disorders and meningeal irritations may occur, depending on the site where larvae are located in the CNS (Sayg1 1999; Liu 2019; Zarlenga and Gamble 2019).

In ophthalmic cysticercosis, the cysticerci are usually located in the retina, anterior, and posterior camera of the eye. These larvae, especially those pressing on the optic nerve, can cause visual field loss and deficits in vision. Larvae that settle in the retina can cause hemorrhage and retinal tears (Sayg1 1999; Liu 2019). In subcutaneous cysticercosis, the cysts resemble small lipomas that can be easily palpated. In muscular cysticercosis, the larvae settle in voluntary muscles and generally do not produce symptoms, as they continue their development. Sometimes they can cause myositis and therefore, eosinophilia and fever.

In addition, swelling, atrophy, and fibrosis may occur in the muscles. However, cysticerci that die in muscle tissue do not cause any symptoms, if they are calcified (Liu 2019; Zarlenga and Gamble 2019). These metacestodes, which cause serious life-threatening diseases in humans, do not cause any significant clinical symptom in their intermediate pig hosts. In some exceptional cases, tongue paralysis and convulsions may be observed in pigs. However, the presence of cysticerci in pigs spoils the aesthetics of the meat and thus has important economic impacts.

\section{Diagnosis}

The most commonly used conventional and reliable method for the diagnosis of mature T. solium in humans is stool examination. For this purpose, the stool is examined macroscopically for discharged proglottids and microscopically for eggs. In microscopic examination, eggs are sought by using flotation methods. However, it is not possible to distinguish Taenia eggs seen in the stool examination at the species level, so the species responsible for the infection cannot be determined by fecal examination. The only known way to determine to the causative species is to obtain the gravid proglottids that were thrown off individually or in groups and to count the uterine side branches in these rings, which are the only known feature for pre-diagnosis. If the scolex can be found after treatment, the presence of four large suckers and hooked rostellum also helps to distinguish the parasite from T. saginata. Serological techniques are used for diagnosis, while more advanced techniques such as PCR are used to differentiate species (Allan et al. 1996; Sayg1 1999; Liu 2019; Zarlenga and Gamble 2019). The definitive diagnosis of cysticercosis can also present difficulties due to the tendency of parasite to settle in brain tissues, where routine biopsy is not possible. For this reason, serological tests of antibodies against cysticercosis and imaging techniques, such as CT, MRI, and $\mathrm{x}$-rays are used in the diagnosis of human cysticercosis (Schantz et al. 1993; Sayg1 1999).

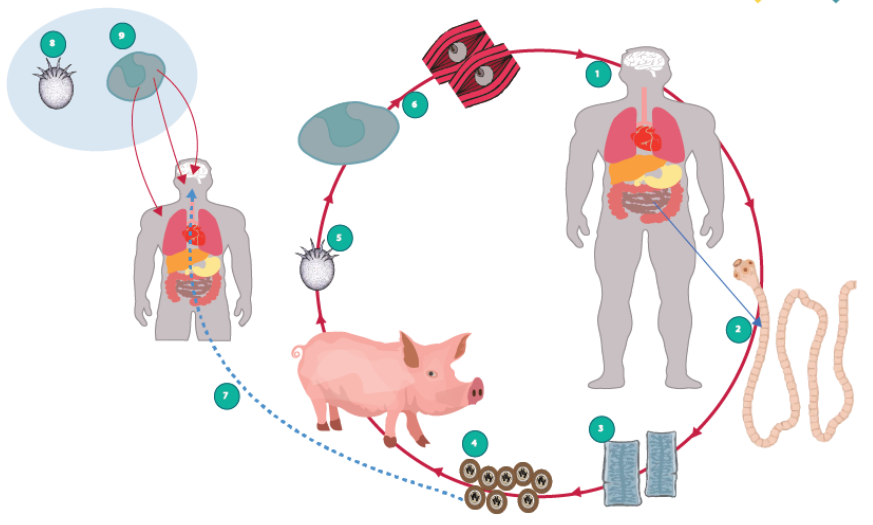

Fig. 1: Life cycle of T. solium (Illustrated by B Senlik)

1: Humans infected by ingesting raw or undercooked infected meat. 2: Adult tapeworm developed and attached in small intestine 3: Gravid proglottids and sometimes eggs in faeces passed in environment 4: Eggs released from proglottids in the environment are taken by intermediate host pigs with contaminated water and food. 5: After the oncospheres inside the egg is released, it gradually settles in the tongue, diaphragm and other muscles of the pigs. 6: In these muscles, they become fluidfilled cysticercus. 7: In humans, infection can also occur by oral ingestion of eggs that have been passed into the environment, or sometimes by the development of eggs in their own intestines as a result of auto infection. 8: In this case, the released oncospheres go to various body parts and settle. 9: The oncospheres, which are located in organs such as eyes, brain, tongue, muscles and under the skin, develop here and form cysticerci.

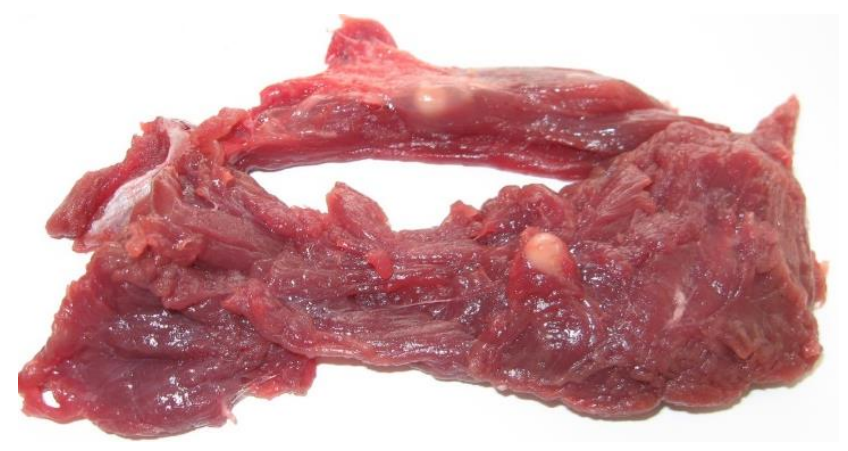

Fig. 2: C. cellulosae in pig muscles (from B. Şenlik).

Calcification of C. cellulosae helps to determine its localization in the central nervous system and muscles. Brain scans, using CT and MRI, can show the lesion and allow a pre-diagnosis before an operation. An x-ray examination of the soft tissue of the extremities can show the characteristic rice grains or calcified shadows, called "puffed rice" (Sayg1 1999). Eye cysticercosis is usually diagnosed by looking at the morphology and movements of the larval form (Sayg1 1999; Liu 2019; Zarlenga and Gamble 2019).

In pigs, the diagnosis of $C$. cellulosae is made by meat examination. The diagnosis can be made in living animals by examining the tongue for cysticerci. However, the reported sensitivity of tongue examination is low. After cutting the meat, the muscles and organs, where the larvae can be found, should be carefully examined by cutting the sections. In particular, the diaphragm, intercostal, heart, tongue, and masseter muscles should be examined for cysticerci. Successful diagnostic results 
have also been obtained by ELISA, using some fractions obtained by purification of cysticercus cyst fluid and excretion products of the parasite.

\section{Treatment}

Niclosamide is the anthelmintic of first choice for the treatment of adult parasites in humans. This drug acts by causing the parasite to separate from the intestinal mucosa by necrosis of the scolex and prevents the parasites from sugar absorption. It is taken orally at a dose of $50 \mathrm{mg} / \mathrm{kg}$ in children and a dose of $2.0 \mathrm{~g}$ in adults. Praziquantel is used orally at a dose of $5-10 \mathrm{mg} / \mathrm{kg}$ as a single dose. If albendazole is preferred, it should be administered orally, $400 \mathrm{mg}$ once a day for three consecutive days. Another option in the treatment of adult parasites is tribendimidine. This drug is also taken orally and is used at a dose of $200 \mathrm{mg}$ at a time in individuals younger than 15 years old, and $400 \mathrm{mg}$ at a time in individuals older than 15 years (Liu 2019; Zarlenga and Gamble 2019).

The treatment of Neurocysticercosis depends on the symptoms of the disease, the location of the infection in the nerve tissue, and the number of cysts. Conservative treatment is preferred in most patients. Albendazole gives better results than praziquantel. Albendazole 10-15 mg $/ \mathrm{kg} /$ day is used for $8-28$ days, while praziquantel is used $50 \mathrm{mg} / \mathrm{kg} /$ day (divided into three doses) for 15 days. Stool proglottids of individuals treated for adult parasites should be sought for three days after treatment. In addition, stool examinations should be performed 30 days and 90 days after treatment, and the eggs of the parasite should be sought (Bruschi and Gomez-Morales 2017; Liu 2019; Zarlenga and Gamble 2019).

Cysticerci that die after anthelmintic treatment can cause inflammatory reactions in the brain. Therefore, corticosteroids should be used in addition to anthelmintic therapy to reduce the inflammatory reaction and increased intracranial pressure. For this purpose, patients should be given 6o $\mathrm{mg}$ prednisone or $6 \mathrm{mg}$ dexamethasone orally once a day. Corticosteroid therapy should be initiated three days before anthelmintic administration and continued until one week after administration (Sayg1 1999; Bruschi and Gomez-Morales 2017; Liu 2019; Zarlenga and Gamble 2019). In addition, anticonvulsant drugs can be used to help treat seizures that may occur. If symptoms persist despite medical treatment and the patient does not improve, surgical treatment should be advised (Liu 2019; Zarlenga and Gamble 2019). Surgical treatment is more beneficial in orbital cysticercosis. In muscular cysticercosis, positive results can be obtained from praziquantel.

It is not possible to eliminate all cysticerci with anthelmintics in pigs that are intermediate hosts. For this reason, anthelmintic treatment is generally not used in pigs.

\section{Control and Prevention}

In order to successfully combat this disease, the life cycle of the parasite between pigs and humans must be broken.
For this purpose, people carrying the parasite should be treated with an appropriate anthelmintic. It should be borne in mind that an infected person can infect thousands of pigs. Especially in the period when large amounts of proglottids are thrown off after treatment, contamination of the environment by human feces should be prevented, and feces and contaminated water should not be used for irrigation of agricultural areas. Having hygienic toilets alone is not enough to prevent the disease. Accordingly, chemical agents that can inactivate the parasite eggs should be used in the areas where the infection is intense. In addition, attention should be paid to personal hygiene and hand hygiene to prevent the ingestion of parasite eggs spread to the environment by humans. Lettuce, parsley, cress or other leafy vegetables and salad ingredients should not be eaten without washing, as they may be contaminated with eggs (Sayg1 1999; Bruschi and Gomez-Morales 2017; Liu 2019; Zarlenga and Gamble 2019).

People should be prevented from consuming pork with $C$. cellulosae. Meat should be meticulously examined, and pig carcasses should be checked by veterinarians for cysticerci after slaughter. If a large number of cysticerci are found in the carcass, the entire carcass must be destroyed. If small numbers are found, consumption should be permitted provided that the cysticerci are subjected to processes that will destroy them. Meat should be cooked at $80^{\circ} \mathrm{C}$ or at high temperature to reach an internal temperature of $56^{\circ} \mathrm{C}$. Keeping meat at $-10^{\circ} \mathrm{C}$ for 14 days also ensures the inactivation of cysticerci in pork (Sayg1 1999; Bruschi and Gomez-Morales 2017; Liu 2019; Zarlenga and Gamble 2019).

\section{T. saginata TAENIASIS}

This cestode species that infects humans is known as "bovine tapeworm." It is widely seen in regions and countries where cattle are raised in open areas. Although cattle are their intermediate hosts, Cysticercus bovis, the metacestode form of the parasite, can sometimes be seen in deer, antelope, buffalo and llama (Liu 2019; Zarlenga and Gamble 2019).

\section{Epidemiology}

T. saginata infections are common in countries and regions, where cattle are raised freely. In addition, the prevalence is high in regions where raw or undercooked beef consumption is high. Exotic diets, which have gained popularity in recent years, encourage people to consume raw meat. Tourism, illegal immigration, and illegal crossings between countries also contribute to the spread of the infection. Another factor driving the spread of infection is the increase in animals and animal products trade between countries (Bruschi and Gomez-Morales 2017; Liu 2019; Zarlenga and Gamble 2019). Parasite eggs, that enter the environment through human feces, are highly resistant to drought and can maintain their infectivity for a long time, even at low temperatures. For example, eggs can remain infective for 154 days in the 

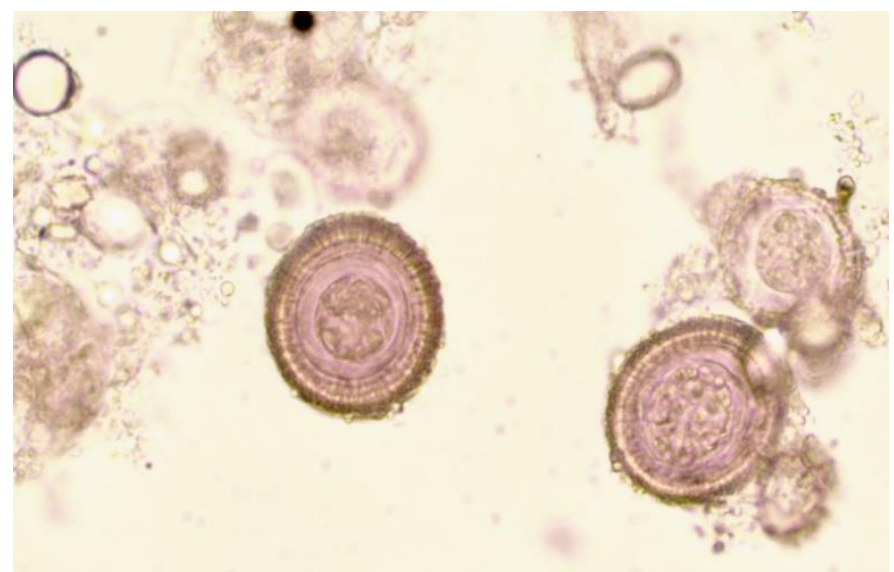

Fig. 3: The appearance of Taenia spp eggs in stool examination (from B. Şenlik).

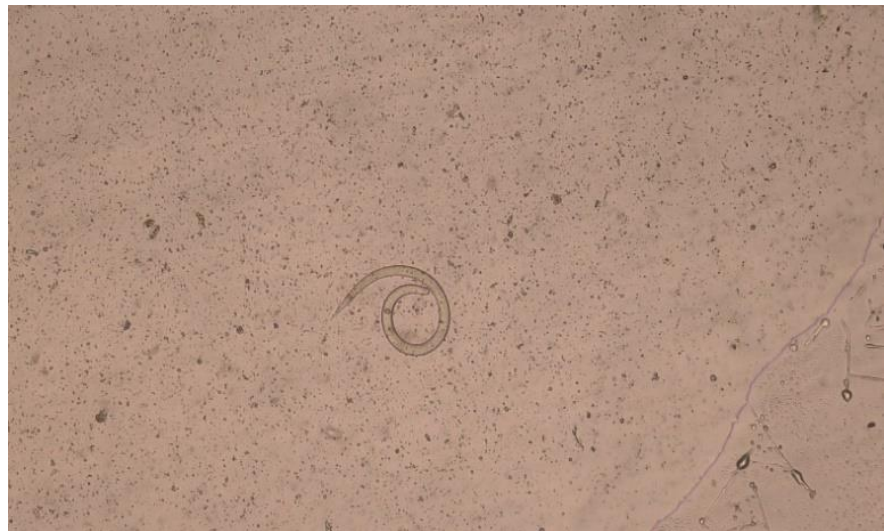

Fig. 4: The appearance of Trichinella spp. larvae in digestion method (from B. Şenlik).

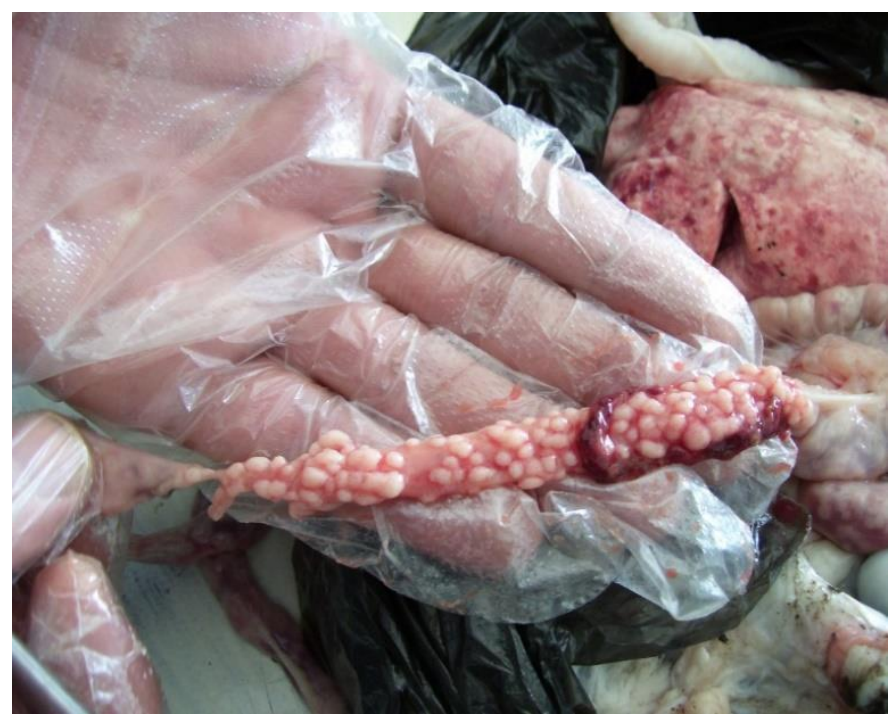

Fig. 5: Sarcocystis cysts in sheep esophagus (from B. Şenlik).

pasture and for more than 70 days in stool. This parasite is common in Africa, the Middle East, Asia, Europe, Latin America, Ethiopia, the Philippines, and Zaire (Liu 2019; Zarlenga and Gamble 2019).
Morphology

The mature form of $T$. saginata is seen in the small intestine of humans, while the larvae form (Cysticercus bovis) is seen in cattle. The length of the parasite varies between 4-12 $\mathrm{m}$ and sometimes can reach $25 \mathrm{~m}$. The strobila bears 1000-2000 proglottids. Each gravid proglottids contains approximately 100,000 eggs. Since the scolex does not contain rostellum and hooks, it is called "unarmed cestod." Cysticerci are 4x6 $\mathrm{mm}$ in size and liquid-filled (Sayg1 1999; Liu 2019; Zarlenga and Gamble 2019). Eggs are spherical or slightly oval and similar in size to other Taenia eggs. It is not possible to distinguish these eggs from other Taenia eggs under the light microscope. Eggs are surrounded by a thin capsule, which disintegrates when eggs leave the proglottids. An average of 720,000 eggs are laid in proglottids per day (Sayg1 1999; Bruschi and Gomez-Morales 2017; Liu 2019; Zarlenga and Gamble 2019).

\section{Transmission}

People are infected by this parasite through eating raw or undercooked beef contaminated with cysticerci, while cattle are infected by ingesting eggs via contaminated feed and water.

\section{Life Cycle}

The final hosts of $T$. saginata are humans, and their intermediate hosts are domestic cattle. However, sometimes animals such as deer, antelope, llamas and giraffes can also be intermediate hosts (Sayg1 1999; Bruschi and Gomez-Morales 2017; Liu 2019; Zarlenga and Gamble 2019).

After T. saginata eggs are ingested by cattle, oncospheres released in the duodenum pass through the blood vessels or lymph channels by piercing the intestinal wall. These eggs circulate through the bloodstream and eventually settle in the tongue, neck, heart, shoulder, and thigh muscles, where they change into the larval form of $C$. bovis. Cysticerci can sometimes develop in organs, such as the lungs, liver, esophagus, brain, and lymph nodes. The development of $C$. bovis begins about 10 days after the oncospheres settle. During this time, cysticerci are seen as small, white lesions with a diameter of 1-2 $\mathrm{mm}$. During the 12-15 weeks after infection, these cysticerci continue to develop and reach a mature length of 6-9 $\mathrm{mm}$. About 8-10 weeks after the eggs are ingested by cattle, these cysticerci become infective and can remain viable in the tissues of intermediate hosts for more than a year (Sayg1 1999; Liu 2019; Zarlenga and Gamble 2019).

Humans are infected by eating the raw or undercooked cysticerci-bearing tissues and organs of intermediate host animals. When these cysticerci in the muscles and organs are eaten by humans, they are separated from the scolex and neck sac by evagination in the jejunum and cling to the intestines with their hooks. While the activity of gastric and intestinal fluids is effective on the evagination 
of the cysticerci in the gastrointestinal system of humans, the speed of the intestinal passage also affects this event. In the development process, proglottids begin to form from the neck area of these young parasites clinging to the intestines. An average of 4-8 proglottids per day form from the neck area and are added to the strobila. Adult cestodes develop 8-10 weeks after the ingestion of cysticerci by humans. Gravid proglottids detached from the strobila fall off the anus due to their own movements. $C$. bovis does not develop in humans except in very rare cases (Sayg1 1999; Bruschi and Gomez-Morales 2017; Liu 2019; Zarlenga and Gamble 2019).

\section{Clinical Signs}

People infected with $T$. saginata usually develop a parasite, but sometimes more than one parasite may develop. In people carrying the adult parasite, the infection is usually mild and asymptomatic. The most common symptom that bothers people the most is the throwing of the proglottids broken off from the parasite's strobila. About 5-10 minutes before the proglottids start to be thrown, the patient feels a sensation in their rectum and the proglottids come out of the anus with a feeling of shivering (Sayg1 1999). The patient may feel uneasy during this process. Proglottids have a higher rate of being thrown during the daytime, when an individual is physically more active. Other clinical symptoms seen in infected individuals include abdominal pain, nausea, vomiting, weakness, weight loss, anorexia, constipation, diarrhea, excitement, and most significantly, anal itching. Abdominal pain is in the form of "hunger pain" and mostly eases after eating (Zarlenga and Gamble 2019). Some patients may also experience dizziness and headache. The severity of the clinical symptoms may vary, depending on the burden of infection (Sayg1 1999; Bruschi and Gomez-Morales 2017; Liu 2019; Zarlenga and Gamble 2019).

Adult $T$. saginata in the intestine causes traumatic and irritative effects. Proglottids detached from the strobila can occasionally cause obstruction in the pancreas and bile ducts or obstruct lumen of the appendix and cause acute appendicitis (Liu 2019; Zarlenga and Gamble 2019).

C. bovis does not usually cause clinical symptoms in cattle. In experimental studies, it was observed that it caused fever and stiffness in the muscles. In addition, it has been reported that glycogen synthesis is negatively affected in the skeletal muscles and liver in infected cattle, and embolism may also be seen (Sayg1 1999).

\section{Diagnosis}

Since $T$. saginata infections in humans do not have a characteristic clinical presentation, diagnosis is based on laboratory findings. Diagnosis can be made by stool examination, the cellophane band technique, serological tests, and searching for coproantigens in the stool (Gottstein et al. 1991). Stool examinations sometimes may be insufficient to reveal infections. Because there is no uterine hole in the proglottids of the parasite, the eggs cannot be released before the proglottids are broken. In such cases, the egg may not be detected in the stool. As in $T$. solium infections, eggs detected in the stool cannot be distinguished by classical examination under light microscopy. Eggs can only be distinguished from one another using molecular techniques (Liu 2019; Zarlenga and Gamble 2019). Although antibodies are formed against this parasite in humans, there is no specific test that can be used for diagnostic purposes. Since $T$. saginata does not cause a life-threatening clinical condition in humans, it is sufficient to detect proglottids in the anal area or in the feces.

The diagnosis of $C$. bovis in cattle can usually be made at a necropsy, or during the meat examination performed in the slaughterhouse (Sayg1 1999; Liu 2019). Examination should start with the masseter muscles. For this purpose, sections should be made parallel to the mandible, then the neck muscles should be examined. Subsequently, the tongue, diaphragm and heart should be examined. It should be kept in mind that cysticerci can be overlooked during normal meat examination in mild infections, since it is not possible to cut all the muscles of the body for examination. For this reason, meat examination should be carried out carefully and meticulously in accordance with rules. Some serological methods, such as ELISA, can be used for diagnosis in cattle. The specificity of the ELISA method with monoclonal antibodies has been found to be very high, and it has been shown that this method allows the detection of infection in cattle with 50 cysticerci (Harrison et al. 1989; Brandt et al. 1992).

\section{Treatment}

Treatment of $T$. saginata-infected people is of paramount importance, as they are a source of infection for cattle in their area (Liu 2019; Zarlenga and Gamble 2019). Anthelmintics, such as niclosamide, praziquantel and albendazole, used for the treatment of $T$. solium infections can be used. Corticosteroids or anticonvulsants are unnecessary in $T$. saginata taeniasis, since cysticerci are not formed in humans. However, in order to reduce complaints such as vomiting and nausea, auxiliary drugs can be used in treatment. Treatment of cattle carrying $C$. bovis is typically not applicable. Especially in cattle with a large number of larvae, it is not possible to destroy all of them. However, when needed, praziquantel and albendazole can be used.

\section{Control and Prevention}

Since people are the main source of infection, stool must be disposed of properly during and after treatment. In the absence of hygienic toilets in underdeveloped countries or regions, the proglottids discarded with feces should be collected and burned in a fire or boiled in water. In addition, contamination of the environment by human excreta should be prevented by installing appropriate sewage systems, and sewage water should not be used for irrigation of agricultural areas (Sayg1 1999; Bruschi and Gomez-Morales 2017; Liu 2019; Zarlenga and Gamble 
2019). Contamination of the pastures grazed by cattle with sewage waste should be prevented. Social activities such as excursions, trekking, camping and picnics enable $T$. saginata eggs to easily spread to the environment. For this reason, infection of the surrounding cattle with $T$. saginata eggs should be prevented by installing hygienic toilets at resting areas, waterfront, picnic, and recreation areas. In addition to these measures, the meat of slaughtered animals should be examined by veterinarians for cysticerci.

Cysticerci die when beef is cooked to an internal temperature of $56^{\circ} \mathrm{C}$ for 20 minutes or when the infected carcass is frozen at $-10^{\circ} \mathrm{C}$ for $10-14$ days. In addition, cysticerci are sensitive to the salt, and become inactivated in meat containing at least $\mathbf{2} \%$ salt. If meat is kept in $\mathbf{2 0} \%$ salt brine for 21 days, cysticerci lose their vitality (Sayg1 1999; Erol 2007; Zarlenga and Gamble 2019).

The habit of eating raw meatballs plays an important role in the spread of the disease. Therefore, if eaten raw, meatballs must be made from sheep meat. Additionally, public awareness should be raised about the transmission routes and importance of the disease.

\section{T. asiatica TAENIASIS}

T. asiatica, which was first detected in indigenous people living in mountainous regions in Taiwan, was initially likened to $T$. saginata (Zarlenga and Gamble 2019). However, later studies revealed that this species is morphologically and genetically different from $T$. saginata (Liu 2019; Zarlenga and Gamble 2019). Although the intermediate host of this species is the pig, a cysticercus is not formed in humans as in $T$. solium infections (Zarlenga and Gamble 2019). This species is also known as "Asian tapeworm” or "Taiwan tapeworm.”

\section{Epidemiology}

Compared to the other two Taenia species, T. asiatica is spread in a more limited area. This parasite can be seen in many Asian countries, such as Korea, Indonesia, Taiwan, Thailand, China, and Japan (Schantz et al. 1998; Anantaphruti et al. 2007; Liu 2019; Zarlenga and Gamble 2019). The intermediate hosts of this species are domestic and wild pigs. Unlike other species, the metacestode form of the parasite prefers the liver and lungs of the intermediate host. Therefore, eating the raw or undercooked internal organs of infected pigs contributes to the spread of the disease (Liu 2019; Zarlenga and Gamble 2019).

\section{Morphology}

The scolex of $T$. asiatica is spheroidal and approximately $0.8 \mathrm{~mm}$ wide. There are no hooks on the rostellum, which has a pointed appearance. The strobila of an adult cestode contains 700-900 proglottids and is approximately $3.5 \mathrm{~m}$ in length. Each gravid proglottids contains an average of 80,000 eggs. The size of the metacestode, $C$. viscerotropica, which develops in the liver of domestic and wild pigs, is smaller than the larvae of other species, measuring up to $2 \times 2 \mathrm{~mm}$ (Liu 2019; Zarlenga and Gamble 2019). The structure of the gravid proglottids is similar to those of $T$. saginata. Similarly, the spherical eggs are similar in shape to the eggs of other Taenia species.

\section{Transmission}

People ingest $C$. visceratropica in the raw or undercooked liver and lungs of pigs. Pigs consume the eggs via contaminated water and feed.

\section{Life Cycle}

The definitive host of this cestode is humans and its intermediate hosts are domestic and wild pigs. While the adult parasite settles in the small intestines of humans, the metacestode form develops in the liver and lungs of intermediate hosts. Experiments have demonstrated that cattle, goats, rodents and monkeys can be infected in addition to pigs (Zarlenga and Gamble 2019). Most of the larvae in the liver settle in the parenchyma in pigs, while some grow on the surface. Experimental studies have also shown that the omentum, colon serosa, diaphragm, abdominal muscles, gall bladder, and peritoneal cavity are alternative locations of larvae. The life cycle is generally the same as that of the other species. C. viscerotropica develops in the liver approximately four weeks after pigs ingest eggs released from proglottids excreted in human feces. Larvae consumed by eating raw or undercooked infected pig liver are evaginated in human small intestines and become adult parasites after 2.5-4.0 months. Adult parasites in infected individuals can live for more than two years (Galán-Puchades and Fuentes 2013; 2014; Liu 2019; Zarlenga and Gamble 2019).

\section{Clinical Signs}

As in infections caused by other Taenia species, clinical symptoms are generally not observed in $T$. asiatica infections. Infected individuals may not be aware that they are carrying the parasite until they start throwing the proglottids. However, in some cases, where the number of parasites is high, clinical symptoms may be apparent. Infected individuals may experience emotional trauma during the exit of the proglottids from the anus, even if they do not show symptoms (Liu 2019; Zarlenga and Gamble 2019). Other than the discharge of the proglottids, other common symptoms include nausea, dizziness, headache, loss of appetite, constipation, hunger, hunger pain in the abdomen, diarrhea, and vomiting. Less frequently, symptoms such as muscle pain, stomach pain, drowsiness, convulsions, anxiety, skin itching, and respiratory disturbance may may be seen (Galán-Puchades and Fuentes 2013; 2014; Liu 2019).

Although necrosis, hepatocyte degeneration, and granulomatous reactions occur in the liver of intermediate host, no typical clinical symptoms of the disease occur in these animals. 


\section{Treatment}

Few treatments exist specifically for T. asiatica. Successful results have been obtained from oral administration of praziquantel in a single dose of $150 \mathrm{mg}$. Anthelmintics, such as niclosamide and albendazole, which are used in the treatment of other Taenia types, can also be used in $T$. asiatica infections (Zarlenga and Gamble 2019).

\section{Control and Prevention}

The best way to prevent this disease is to avoid eating pig livers and viscera contaminated with $C$. viscerotropica. As in $C$. cellulosae infections, $C$. viscerotropica from the viscera of hunted animals or domesticated pigs should be inactivated by high temperature cooking or freezing. In addition, infected people should be treated and feces should be prevented from contaminating the environment.

\section{TRICHINELLOSIS}

Trichinellosis can be transmitted among mammals and humans, and may be fatal. Eating raw or undercooked meat of pigs, bears, horses, seals and other omnivorous animals infected with Trichinella spiralis and various species of the Trichinellidae family transmits the disease to humans (Gajadhar et al. 2009; Bruschi and GomezMorales 2017; Zarlenga and Gamble 2019; Pozio and Bruschi 2019).

T. spiralis was first identified by Baget during an autopsy in England in 1835. The first clinical case of Trichinellosis was reported by Zenker in 1860 , when larvae inside a capsule were detected in the arm muscles of a young girl, who died of a typhoid-like disease (Zarlenga and Gamble 2019).

\section{Etiological Agents}

The genus Trichinella is a complex, consisting of 12 species and many genotypes spread over a wide geography. Trichinella species, which cannot be morphologically distinguished from one another, are divided into two groups on the basis of their ability to form a capsule in the host muscles. The species encapsulated in the host muscle cells are T. spiralis, $T$. nativa, T. britovi, T. murrelli, T. nelsoni and Trichinella T6, T8, T9 and T12. Species that do not form capsules in host muscle cells are $T$. pseudospiralis, $T$. papuae and $T$. zimbabwensis. Encapsulated Trichinella species infect mammals. Among the unencapsulated species, $T$. pseudospiralis infects mammals and birds, while $T$. papuae and T. zimbabwensis are also seen in mammals and reptiles (Gajadhar et al. 2009; Pozio and Bruschi 2019; Zarlenga and Gamble 2019).

\section{Epidemiology}

Human infections are caused by eating raw or undercooked meat of animals that harbor larvae in their muscles, especially pigs. In addition, meat products prepared from the meat of these animals also contribute to contamination. Trichinellosis, a highly dangerous zoonosis, is estimated to affect up to 11 million people worldwide (Dupouy-Camet 200o). Infections are widespread, especially where there is little or no control of rodents such as mice and rats, animals are not under the control of a veterinarian, pigs are fed with animal tissues, or animal carcasses are discarded randomly. Transmission between animals occurs by eating meat infected with Trichinella larvae as a result of cannibalism or carnivorism. Wild mammals in nature act as reservoirs for these parasites. These animals are important in the transition of infections from the rural to the domestic cycle. The role of carnivorous birds and reptiles, such as crocodiles and lizards, in the natural cycle of these parasites is not yet fully understood (Pozio and Bruschi 2019). In the epidemiology of the disease, mice are the main carriers. These animals eat carrion or each other, causing the infection to continue in mouse colonies.

Trichinella species can be seen in humans and in many animal species. This nematode can infect more than 150 mammal species, such as domestic or wild boar, rat, mouse, bear, horse, seal, walrus, dog, fox, jackal, and wolf, but is rarely seen in birds. Trichinella species have been detected in domestic animals, mainly pigs, in 43 countries, and in wild animals in 66 countries. Trichinellosis has been reported in humans in 55 countries worldwide. On the other hand, there is no information about Trichinella infections in 92 countries (Pozio and Bruschi 2019). Trichinella infections have been found in domestic and wild pigs in Germany, Austria, France and Italy. While the larvae are seen in striated muscles, adult forms are embedded in the small intestine mucosa. Both the mature form and its larvae can be found in the same host (Rommel et al. 200o).

The distribution of the species varies according to geographical regions and countries, with some species are seen in some countries to a limited extent. For example, the spread of T. nelsoni is limited to Southeast Africa. T. spiralis and $T$. pseudospiralis are cosmopolitan species. Serious clinical symptoms, and even death, were seen in the first human case of T. pseudospiralis in 1993 (Campbell 1988; Macpherson et al. 200o).

As a zoonotic disease for about 150 years, Trichinellosis has seriously threatened public health in both developed and developing countries. In the European Union countries, 420 million dollars are spent for the mandatory examination of 190 million butchered pigs each year, and 1 billion dollars is spent for operations, such as freezing and cooking meat in the US (Pozio 1998).

Trichinella species are the largest known intracellular parasites. Among the Trichinella species, T. spiralis is the most known and the most common type of infection. Larvae of this species are encapsulated in fibers of striated muscles and preserve their vitality for years. Larvae can survive for 39 years in humans and 11 years in pigs.

Transmission

Pigs become infected by eating infected mice, other animals, or trichinated pig waste. Cannibalism between 
pigs also causes the spread of the disease. Animals such as sheep, cattle, and horses can be a source of infection due to the use of animal proteins as a feed for grass-eating animals (Rommel et al. 200o).

The main source of human infections is pork infected with larvae. Larvae in pigs are commonly found in the diaphragm, tongue, masseter, and intercostal muscles. Raw and insufficiently cooked pork and pork sausages are seen as the most important causes for the spread of the infection. Human consumption of the never frozen, raw, or undercooked meat of animals such as horse, bear, walrus, dog, or fox can also lead to infection (Prasad 2010).

\section{Life Cycle}

Adults of the genus Trichinella settle in the small intestine, while the infective larvae settle in the muscles of the same host. These parasites do not have a free life stage.

When the meat of infected animals is eaten by another host, L1 larvae are released in a few hours with the help of digestive enzymes in the stomach and pass into the small intestine. Here, they become adults in 2-4 days. However, if the host has a hypersensitivity to the parasite or diarrhea, the larvae in the small intestine, as well as the adults that have developed, will be excreted with feces. Reinfection can occur if food contaminated with feces containing larvae or adult parasites excreted in this way are eaten by a carnivorous, omnivorous, or herbivorous animal (Gajadhar et al. 2009; Bruschi and Gomez-Morales 2017; Pozio and Bruschi 2019; Zarlenga and Gamble 2019). Adult parasites in the small intestine of the host settle among the villi. The male and female parasites mate, and then the males die. Females enter the Lieberkühn glands and Peyer's patches, and three days after mating begin to give birth to Li larvae. A female can give birth to 10oo10,0oo larvae during her life time. These larvae enter the lymph channels and come to the left vena cava cranialis through the ductus thoracicus, and then spread to all organs and tissues via circulation. Larvae generally prefer muscle groups with high blood flow, so they mostly settle in the diaphragm, tongue, larynx, eye, masseter, intercostal, abdominal, and hip muscles. Although larvae can be found in the liver, pancreas, and kidneys, only the larvae that go to the striated muscles survive, and those that go to other organs and tissues die. Li larvae that reach the striated muscles develop rapidly by settling in the sarcolemma of the muscle fibers. If the infection has occurred with a capsule-forming species, the larvae are encircled by a capsule formed by tissue histiocytes in the area (Pozio and Bruschi 2019; Zarlenga and Gamble 2019). Later, they develop within the capsule and reach a characteristic size and appearance. The capsule, which was previously open at both ends, closes completely and surrounds the larvae. These events are completed in the seventh week of infection and the larvae become infective.

In the later stages of the infection, the muscle fibers with the capsule degenerate and these foci with the larvae begin to become calcified after 5-6 months. However, despite this, the larvae can survive in the striated muscles for years by resisting the immune response of the host. Even after the host dies, the larvae can survive for a long time. Larvae that have settled in muscle cells do not show any further development and continue their cycle when the meat containing larvae is eaten by another host (Gajadhar et al. 2009; Bruschi and Gomez-Morales 2017; Pozio and Bruschi 2019; Zarlenga and Gamble 2019).

\section{Clinical Signs in Humans}

An incubation period begins with the ingestion of Trichinella larvae by humans, and symptoms appear at the end of this period. The incubation period varies from 2 to 45 days, depending on the type of meat consumed (raw or undercooked), the consumption frequency, the number of live larvae ingested, the region where the larvae are settled, the type or genotype of the parasite, and host immunity. A short incubation period indicates that the prognosis of the disease is poor. The incubation period is usually short in severe cases of Trichinellosis (Pozio and Bruschi 2019).

In the first week of the infection, gastrointestinal symptoms, such as hyperemia in the small intestines, catarrhal enteritis and minor wounds, nausea, vomiting, diarrhea, and abdominal pain are observed. Stool is mostly soft, greenish-brown in color, and sometimes contains mucus. However, there is no blood. Electrolyte imbalance and hypoproteinemia occur in patients due to fluid loss. One week later, edema occurs on the eyelids and face along with persistent headache, dizziness, and insomnia. Edema sometimes covers the whole face (Pozio and Bruschi 2019; Zarlenga and Gamble 2019). In severe cases, edema can spread to arms and legs. Symmetrically shaped edema heals within 5-6 days after corticosteroid treatment. In addition, hyperemia and bleeding occur in the host, and eye itching and lacrimation are observed. Patients with such symptoms typically avoid light. Other common symptoms of the disease are severe sweating and high fever $\left(40-41^{\circ} \mathrm{C}\right)$. Sometimes, fever can take up to three weeks depending on the severity of the infection. Tachycardia occurs due to the increase in body temperature. In addition, cough, pulmonary edema, and diaphragm pain occur in relation to the respiratory system. The patient may experience dehydration, weakness, and cachexia, as well as dizziness, headache, and ascites (Gajadhar et al. 2009; Pozio and Bruschi 2019; Zarlenga and Gamble 2019).

Following these symptoms, patients may experience muscular pain, which is especially seen in the neck, arms, and legs. Therefore, the patient does not want to move. In addition, the pain can manifest itself severely in the diaphragm, masseter, intercostal, and orbital muscles. Myalgia continues for 2-3 weeks. In severe infections, fatalities may occur as a result of paralysis of the respiratory muscles, especially the diaphragm. Eosinophilia of $60-85 \%$ is seen in patients and leukocytosis may develop. 
In severe cases, complications related to various tissues and organs, such as encephalitis, myocarditis, dizziness, visual impairment, and dyspnea, occur within the first two weeks. In mild and moderate cases and in patients who are not treated appropriately, there is a possibility of complications in later periods (Pozio and Bruschi 2019; Zarlenga and Gamble 2019).

Chronic Trichinellosis can develop after acute cases that are not treated well or leave sequelae. In such patients, pain may not go away, quality of life may be impaired, and paranoia may occur (Pozio and Bruschi 2019).

\section{Clinical Signs in Animals}

Trichinellosis is generally mild and does not cause clinical symptoms in animals. In severe infections, adult parasites can cause enteritis, while larvae reaching the muscles can cause acute myositis, fever, and eosinophilia (Macpherson et al. 2000).

\section{Diagnosis}

Although eosinophilia, leukocytosis, and sediment increase are helpful in diagnosis, definitive diagnosis is not possible in live animals. However, a diagnosis can be made by examining muscle samples taken from suspected animals during meat examination in slaughterhouses.

Two main methods are used for the direct detection of Trichinella in the meat: trichinoscopy and the digestion method (Şenlik 2011). The appropriate method must be chosen to get reliable results. For example, there is no capsule around T. pseudospiralis and T. papuae. Species without capsules are very difficult to detect by trichinoscopy. For this reason, all meat samples should be examined by both methods (Şenlik 2011). Trichinella larvae prefer different muscles in different animal species. Therefore, samples should be taken from these muscles for both routine meat examination and epidemiological studies. Samples should be taken from the tongue and diaphragm muscles of animal species in which the preferred locations of larvae are unknown (Şenlik 2011).

For this reason, the amount of sample that should be taken varies according to animal species. In addition to these two methods, the DNA of the parasite can be detected in meat samples by multiplex PCR (Pozio and Bruschi 2019).

Differential diagnosis is important for appropriate and timely treatment in humans. Fever with orbital and facial edema can occur in cases of glomerulonephritis, dermatomyositis, allergic reactions, or intoxications. In addition, neck stiffness, headache, confusion, and diseases with excessive arousal should be ruled out. Even in atypical cases, with high fever and eosinophilia but without specific findings, other parasitic diseases such as Fasciolosis and Toxocariosis should be considered. Serological tests can also be used to diagnose Trichinellosis cases in humans. ELISA used for this purpose shows a high sensitivity and specificity. The definitive diagnosis of the disease in humans is made by examining biopsy samples by parasitological and histological methods, preferably taken from the deltoid muscles. If this is not possible, a o.2-0.5 g muscle sample should be taken from any part of the body. The muscle samples can be examined by trichinoscopy or the digestion technique, or multiplex PCR can be used to identify the DNA of the parasite (Pozio and Bruschi 2019; Zarlenga and Gamble 2019).

\section{Treatment}

Since definitive diagnosis cannot be made in living animals, treatment is not possible in usual practice. However, in experimental studies, anthelmintics, such as mebendazole, flubendazole, albendazole, fenbendazole, cambendazole, and thiabendazole, were found to be effective against both adult and larval stages of the parasite. Flubendazole is effective against both larvae and mature parasites when administered by adding to pig feed for two weeks at doses of 30-125 ppm. Febantel is effective at a dose of $20 \mathrm{mg} / \mathrm{kg}$ and albendazole at a dose of 10 $\mathrm{mg} / \mathrm{kg}$ against larvae (Rommel et al. 2000).

In humans, benzimidazole compounds are used against parasites and corticosteroids are used to eliminate symptoms. Mebendazole is the most commonly used benzimidazole. Anthelmintics should be used within one week of infection to destroy adult parasites in the intestines and to prevent the larvae from spawning and going to the muscles. However, it is not possible to determine when the infection started. Therefore, in practice, mebendazole or other anthelmintics should be used within 48 hours after the suspected meat has been eaten. Mebendazole is used at a total daily dose of 5 $\mathrm{mg} / \mathrm{kg}$ and divided into two doses. The drug should be used for 10-15 days when treatment is started after the clinical picture has taken shape, then a 5-day break should be taken. Then it should be used again for 10-15 days. This treatment protocol should be continued for 4-6 weeks. Mebendazole can also be used at a dose of 20-25 $\mathrm{mg} / \mathrm{kg}$ in treatment. However, it should be kept in mind that allergic reactions may occur and liver enzymes may increase when used in high doses (Pozio and Bruschi 2019). Mebendazole should not be used in pregnant women in the first trimester. Pyrantel at a daily dose of 10 $\mathrm{mg} / \mathrm{kg}$ and levamisole at a dose of $2.5 \mathrm{mg} / \mathrm{kg}$ against adult parasites are also effective (Pozio and Bruschi 2019).

In addition to anthelmintic therapy, corticosteroids can be used to alleviate patients' symptoms and accelerate recovery. For this purpose, prednisolone can be administered with a daily dose of 30-6o mg for 10-14 days (Pozio and Bruschi 2019).

\section{Control and Prevention}

As mentioned earlier, pigs are the main source of Trichinellosis in humans. People become infected through eating raw or undercooked pork or meat products containing pork, such as salami and sausages. In certain parts of the world, wild pigs, polar bears, various seal species, horses and some fur animals are also blamed for human Trichinellosis. 
Preventive measures that can be taken on pig farms include separating dead animals from the herd immediately, ensuring no garbage is kept near the farm, controlling rats, and informing consumers when necessary (Gajadhar et al. 2006).

The most critical point in control is the inspection of pork meat before sending to market. The methods identified in the diagnosis section should be used to determine whether meat is infected. If positive samples are found as a result of the tests, the carcasses must be destroyed. Epidemiological screening should be used at farms, where pigs with positive muscle samples originated to identify other infected pigs, which should be removed from the herd (Gamble 1998; Gamble et al. 1996). High temperature cooking, as well as freezing of meat, have a lethal effect on the larvae in the muscles. Thus, meat must be subjected to the following procedures before being offered for consumption in endemic regions (Pozio and Bruschi 2019; Zarlenga and Gamble 2019). The main inactivation processes that can be used for this purpose are as follows.

\section{Cooking}

Cooking the meat at $77^{\circ} \mathrm{C}$ for at least 10 minutes inactivates the larvae (Erol 2007).

\section{Freezing}

Cold shocking should be done in deep freezers, so that the internal temperature of meat is $-40^{\circ} \mathrm{C}$. Different freezing procedures are used in different countries and regions. For example, in the United States, meat that is 15 $\mathrm{cm}$ thick is kept at $-15^{\circ} \mathrm{C}$ for 3 weeks, while meat that is 69 $\mathrm{cm}$ thick is kept at $-15^{\circ} \mathrm{C}$ for 4 weeks. On the other hand, in European Union countries, meat is frozen at $-25^{\circ} \mathrm{C}$ for 10-20 days, depending on its thickness. However, not all species are inactivated by freezing. For instance, T. nativa, which causes Trichinellosis in polar bears, is resistant to freezing. It has been reported that meat retains its infectivity even months after freezing (Erol 2007).

\section{Irradiation}

In countries where this method is permitted, gamma rays at a dose of $0.3 \mathrm{kGy}$ can be applied (Erol 2007).

\section{TOXOPLASMOSIS}

Toxoplasmosis is one of the most important parasitic zoonoses of humans and can be seen in almost every part of the world. The disease is caused by Toxoplasma gondii, an obligate intracellular protozoan.

\section{Hosts}

The final host of the parasite is domestic and wild cats. Many warm-blooded animal species serve as intermediate hosts, including poultry and humans. T. gondii is found inside the cell in muscle tissue and organs such as the heart, brain, and liver of humans and many intermediate host animals such as sheep, goat, cattle, pigs, and chickens (Dubey and Beattie 1988).

\section{Epidemiology}

In terms of human and veterinary medicine, $T$. gondii is a very important protozoonosis, widely seen in humans and animals all over the world (Dubey and Beattie 1988; Fayer 1994). The disease can be found in all continents except Antarctica. It is estimated that 500 million people worldwide are infected with $T$. gondii, although they may be asymptomatic. More than $50 \%$ of women of childbearing age are reported to be infected with Toxoplasmosis in Western Europe, Africa, South America, and Central America. Seropositivity rate of Toxoplasmosis among adults in Europe ranges from 10-80\%, and in Central Europe it ranges from $30-45 \%$ in women of childbearing age. In HIV patients, the prevalence is higher, between 50-75\% (Fayer 1994; Parija 2009). The prevalence of this disease, which is more common in animals intended for slaughter, can reach up to $90 \%$ in some regions.

The prevalence of $T$. gondii infections in humans and animals varies by country, and even among different geographic regions of the same country. Many factors, such as feeding habits, animal breeding systems, hunting, culture, and cleaning habits, affect the spread of the disease.

Environmental factors may also affect the spread of $T$. gondii infection. The rate of infection is higher in hot climates and regions with low humidity, while infection is less common in cold climates and mountainous and dry areas. For example, in Latin America and Africa, which are very suitable for the development of oocysts, the prevalence of the disease can reach up to $90 \%$.

\section{Morphology}

Non-sporulated oocysts excreted with feces in cats are $10 \times 12 \mu \mathrm{m}$ in diameter, while the diameter of sporulated oocysts is $11 \times 13 \mu \mathrm{m}$. Sporocysts are $6-8 \mu \mathrm{m}$ in size. The tachizoids found in the intermediate host are crescent shaped and range in size from $2 \times 4$ to $4 \times 8 \mu \mathrm{m}$. The size of the bradyzoites found in the tissue cysts of intermediate hosts is $1.5 \times 7 \mu \mathrm{m}$. Young cysts are $5 \mu \mathrm{m}$ in size, while those in the brain can reach up to $70 \mu \mathrm{m}$. The length of the cysts in the muscles can reach $100 \mu \mathrm{m}$. The thickness of the wall of the cyst, which is not divided into sections, is less than $1 \mu \mathrm{m}$ (Dumanlı and Aktaş 2015).

\section{Transmission}

The most important reservoirs for human infections are livestock infected with Toxoplasmosis. Transmission occurs by oral ingestion of any of the infective forms of the parasite, such as tachizoid, bradyzoite, or oocyst (Parija 2009). Humans become infected by (1) eating raw or undercooked meat of intermediate hosts such as sheep, goat, or pigs that contain tachizoids or bradyzoites; (2) by 
ingesting sporulated oocysts obtained by contact with cats via drinking water or food; or (3) congenitally. Intermediate host livestock or other animals take the disease by ingesting feed or water contaminated with sporulated oocysts shed by cats. Cats become infected by eating tachizoids and bradyzoites found in the tissues of intermediate hosts, infected organs and meat, and by ingesting sporulated oocysts with contaminated food and water.

\section{Life Cycle}

Cats, which are the final hosts, can also be intermediate hosts. After infective forms ingested by cats enter the intestinal epithelium, they pass the periods of Merogony and gametogonia and produce oocysts within 3-10 days. In infected cats, oocysts are excreted with feces for 1-2 weeks. These oocysts, which are shed to the outside environment without sporulation, sporulate in a short time in environments with suitable temperature and humidity (Joynson and Wreghitt 2005; Shakespeare 2009; Dumanlı and Aktaş 2015). The sporulated oocysts, taken up by the intermediate hosts, are broken down by digestive enzymes and the sporozoites are released. Later, these sporozoites go to cells of reticuloendothelial system (RES), become tachizoids and multiply to form pseudocysts. When the tachizoid number reaches 8-16, the cell disintegrates and infect new cells. This period is the acute form of the infection. Tachizoids released from pseudocysts grow and multiply in endothelial cells by going to various organs and forming tissue cysts containing bradyzoites. These cysts are mainly found in the brain, heart, skeletal muscles, eye and, to a lesser extent, other organs and tissues. Thousands of bradyzoites can be found in these cysts, which can reach $100 \mu \mathrm{m}$ in diameter. The host cannot resist these cysts with an adequate immune response, so they can survive in intermediate hosts for many years or even for life. In some intermediate hosts such as cattle, these cysts lose their vitality by calcification after a certain period of time. People and carnivorous animals, that eat the meat of intermediate hosts infected in this way, also become infected (Dumanlı and Aktaş 2015).

Carnivorous animals are infected by eating various tissues and organs of intermediate hosts that contain tachizoids and bradyzoites, as well as by drinking water or eating food contaminated with oocysts. Herbivorous animals such as sheep, goats, and cattle are infected by ingesting oocysts with foodstuffs such as weeds, hay, fodder, and water. Cats become infected by eating the tissues and organs of intermediate hosts carrying tachizoids and bradyzoites or by hunting and eating animals such as mice and rats. Cats can also become infected when they ingest oocysts with contaminated food (Urquhart et al. 1996; Dominique et al. 20o8; Prasad 2010). Tissue cysts can survive throughout the life of the intermediate host. Tachizoids found in body secretions, such as milk, cannot survive long in the external environment. On the other hand, oocysts, the non-host form of the parasite, can survive for 2-3 weeks in the external environment under appropriate humidity and temperature (Urquhart et al. 1996; Dominique et al. 2008; Prasad 2010).

Although sheep and pork pose a high risk for human infections, beef and poultry meat have a lower risk of infection (Dubey 1986; Dubey et al. 1986). In humans or animals with low immunity, the risk of Toxoplasmosis is very high and infection progresses more seriously.

\section{Clinical Signs}

Clinical symptoms that occur in infected humans and animals are quite different. Sometimes no symptoms occur, while sometimes several clinical pictures can be encountered.

\section{Clinical Signs in Humans}

The disease is asymptomatic in more than $80 \%$ of healthy individuals with strong immunity. However, in people with weakened immunity, the disease is severe and sometimes death can occur. Symptoms usually occur within 7-14 days of ingestion. The disease can affect the functions of many vital organs, such as the brain, eyes, liver, lungs, and heart (Hohlfeld et al. 1989; Prasad 2010; Ortega 2019; Souza and Damatta 2019). In symptomatic cases, high fever, cervical lymphadenopathy, retinochoroiditis, sore throat, fatigue, and chronic muscle aches can be seen.

Toxoplasmic encephalitis usually occurs in individuals with suppressed or weakened immunity, as in patients with HIV. The most common symptoms in such patients are high fever, headache, memory loss, dementia, slowed psychomotor movements, and behavioral changes (Hohlfeld et al. 1989; Prasad 2010; Souza and Damatta 2019). In the ocular form of the disease, intraocular pressure increases, and inflammation and blurred vision may occur.

Pregnant women are always at risk for Toxoplasmosis. Infections that occur in the first and second trimesters of pregnancy may result in abortion and stillbirth, and congenital sequelae may occur in the fetus. If the infection is acquired in the third trimester, normal delivery may occur in patients without acute symptoms, but acute chorioretinitis may develop as the child grows. In addition, congenital Toxoplasmosis can cause blindness, mental retardation, multiple organ failure, and hydrocephalus (Hohlfeld et al. 1989; Prasad 2010).

\section{Clinical Signs in Animals}

Toxoplasmosis is generally asymptomatic in animals, though it may cause pregnant animals to abort (Dhaliwal and Juyal 2013; Dumanlı and Aktaş 2015). Sheep are the intermediate hosts mostly affected by Toxoplasmosis in clinical and economic terms. If contamination occurs during pregnancy in sheep that were not previously infected, tachizoids pass to the offspring through the placenta and cause congenital Toxoplasmosis. Infections in the early stages of pregnancy (up to 45-55 days) result in the death of the fetus with abortion. However, since 
the fetus is very small, the abortion is often not noticed. If the infection occurs towards the middle of pregnancy (up to the goth day), abortion occurs. If the dead fetus is not discarded, it becomes mummified in the uterus. If the infection occurs in the last stage of pregnancy (up to the 12oth day), the offspring can be born and appear normal, or may be born weak and thin and die shortly thereafter (Şenlik 2017). Clinical signs are rarely seen in cats. The main symptoms seen in symptomatic cats include enteritis, pneumonia, enlargement of mesenteric lymph nodes, encephalitis, and chronic interstitial nephritis (Dumanlı and Aktaş 2015).

\section{Diagnosis}

Early diagnosis is of great importance in pregnant women, immunocompromised individuals, and patients with chorioretinitis. Various serological tests, molecular methods, and rapid commercial kits can be used for the diagnosis of Toxoplasmosis in humans. The most commonly used serological tests for diagnosis are the dye test, IgG avidity test, ELISA, agglutination, indirect hemagglutination, and indirect fluorescent antibody test (Urquhart et al. 1996; Joynson and Wreghitt 2005; Ortega 2019; Souza and Damatta 2019. Although serological tests are very effective in revealing prenatal infections, they can sometimes be insufficient. In such cases, the diagnosis can be confirmed by performing PCR on blood samples. Molecular methods can detect the DNA of a single tachizoid. Apart from serological and molecular methods, imaging methods, such as CT and MRI, can be used. Although the sensitivity of these methods is low, they can reveal calcifications and hydrocephalus in the brain, especially in congenital Toxoplasmosis (Souza and Damatta 2019).

Many direct and indirect methods are used for the diagnosis of Toxoplasmosis in intermediate host animals. In direct diagnosis, crust, exudates, and smears made from the internal organs of the fetus are prepared, stained with Giemsa and examined under the microscope. Diagnosis can also be made through histological examination of sections of the internal organs of fetus under microscope. White colored focal necrosis of $2 \mathrm{~mm}$ diameter in cotyledons of the fetal membranes discarded in sheep abortions are pathognomic for Toxoplasmosis. In indirect methods, antibodies developed against the parasite or antigens of the parasite are sought. In addition, molecular methods, such as PCR, can be used. Oocysts can be searched for by examining feces in cats by the flotation method or by using rapid commercial kits. However, it is very difficult to distinguish oocysts detected in stool examination from other protozoan oocysts, such as Hammondia (Prasad 2010; Dhaliwal and Juyal 2013; Şenlik 2017).

\section{Treatment}

The easiest way to control the disease in humans is to treat infected individuals. However, implementing control measures worldwide is difficult. Currently available drugs are only effective on tachizoids. Tissue cysts are less sensitive to existing drugs due to their metabolic activities (Urquhart et al. 1996; Ortega 2019; Souza and Damatta 2019). Pyrimethamine and sulfadiazine are generally preferred in the treatment of acute Toxoplasmosis infections. These drugs act by disrupting folic acid synthesis in the parasite. However, these drugs may have toxic effects on host cells and disrupt folic acid synthesis. Other protein synthesis inhibitors that can be used for treatment are spiramycin, clindamycin and clarithromycin. Spiramycin is preferred in the treatment of pregnant women because it passes through the placenta at a lower rate (Souza and Damatta 2019).

In animals, there is no effective treatment for the disease. Therefore, control measures are essential. However, experimental studies have shown that spiramycin, piritrexin, roxithromycin, clindamycin, cyclosporin A, atovaquone, azithromycin, ponazuril and triazine may be effective (McCabe 2001).

\section{Control and Prevention}

The immune system of the host plays the most important role in Toxoplasmosis infections. Although there are usually no symptoms in people with a normal immune system, the disease progresses and can result in death in individuals with a weak or suppressed immune system. Especially in cancer and AIDS patients, Toxoplasmosis has a very severe course and can be fatal. For this reason, pregnant women and individuals with a weak immune system should not have close contact with cats or cat litter and should avoid contact with soil and raw meat. Children should be prevented from touching cat feces in playgrounds and parks (Dhaliwal and Juyal 2013).

In order to prevent contamination from infected meat, it must be subjected to various processes before consumption, as described below:

\section{Cooking}

Raw or undercooked meat, especially raw meatballs, should not be consumed. Meats should be cooked at least 3-5 minutes at the temperature of $66^{\circ} \mathrm{C}$ (Dubey et al. 1990). In addition, tasting meat while making homemade sausages or salami, or while seasoning it, should be avoided.

\section{Freezing}

Freezing meats at $-12{ }^{\circ} \mathrm{C}$ kills tissue cysts. Even freezing meat overnight in a domestic freezer is sufficient to inactivate most of the tissue cysts in the meat.

\section{Irradiation}

Irradiation at 50 krads or high-pressure processing at 400 MPa are effective in killing tissue cysts (Dubey and Thayer 1994). 


\section{Water sanitation}

Surface water that has not been subjected to any treatment should not be used for drinking and washing vegetables and fruit. Water obtained from streams, rivers, lakes, and rivers should never be used as drinking water without boiling (Erol 2007).

The following prevention methods should also be used.

i) To prevent infection of zoo animals with $T$. gondii, brooms, shovels, and all equipment used should be autoclaved or heated to $70^{\circ} \mathrm{C}$ for at least 10 minutes.

ii) Cats are the key animals in preventing Toxoplasmosis in intermediate hosts, as cats are the final hosts of the parasite. Cats should not be given raw meat and offal, and they should not be permitted to hunt and feed on birds, mice, and rats (Şenlik 2017).

iii) Other measures that can be taken regarding cats are as follows:

- Cat feces should be removed daily.

- Cat litter, sand, and feces collected for disposal should never be kept in the kitchen.

- Containers in which cats defecate should be washed thoroughly with hot water after cleaning and disinfected at least once a week.

- Cats should be prevented from contaminating parks and lawns where children play.

- Cats should be prevented from entering the feed stores of other animals and pets.

There is not yet any vaccine available for use in humans. A vaccine called "Toxovax," which was developed to prevent the infection in sheep, is used in England, France, and New Zealand. This vaccine is a live vaccine containing tachizoids produced in cell culture and provides protection for 18 months after administration (Dubey 1998; Şenlik 2017; Souza and Damatta 2019).

\section{SARCOCYSTOSIS}

\section{Etiological Agent}

The final hosts of the Sarcocystis species are cats, dogs, and humans. Its intermediate hosts are mammals, poultry, reptiles, and humans. Humans are intermediate hosts for some species and definitive hosts for others. Sarcocystis species, which are widely seen in the world, show a heteroxene development. There are about 200 species in the Sarcocystis genus that can infect mammals, poultry, reptiles, and fish (Sing et al. 2019). However, only two of them are zoonotic and use humans as the final host. These species are Sarcocystis hominis and Sarcocystis suihominis.

\section{Morphology}

In Sarcocystis species, the size of the oocysts varies from 7-18 x 15-30 $\mu \mathrm{m}$ and their walls are quite thin. The size of the sporocysts ranges from 8-10 x 15-19 $\mu \mathrm{m}$. There are 2 sporocysts inside the oocysts and 4 sporozoites in each sporocyst. The length of the banana-shaped sporozoites is 7-10 $\mu \mathrm{m}$. The size of tissue cysts can vary from a few millimeters to a few centimeters, depending on the species and the intermediate host. Some, called macrocysts, are quite large and can be seen with the naked eye. Others, called microcysts, are very small and cannot be seen with the naked eye. The interior of mature cysts is divided into compartments and is filled with 14 $\mu \mathrm{m}$ long banana-shaped bradyzoites (also called cystozoites) (Sevgili 2015).

\section{Transmission}

Sarcocystis species need two hosts to complete their development. Definitive hosts become infected by eating the raw or undercooked meat of intermediate hosts, such as cattle and pigs, which contain Sarcocystis cysts. Intermediate hosts become infected by orally ingesting oocysts and sporocysts shed with the feces of the definitive hosts (Fayer 2004; Sing et al. 2019).

\section{Life Cycle}

Oocysts or sporocysts shed by the final host (cats, dogs, and humans) arrive in the small intestine after ingestion by the intermediate host, a herbivorous or omnivorous animal. The sporozoites released there migrate to intestinal epithelial cells. The sporozoites then enter the capillary endothelial cells. After the merogony period, a large number of merozoites are formed. The firstgeneration merogony period takes place 15-16 days after the sporocysts are ingested. Merozoites formed as a result of first-generation merogony enter the venous and arterial cells of the internal organs and pass the secondgeneration merogony period there. Merozoites formed as a result of second-generation merogony enter the bloodstream and go to various organs and tissues. The most preferred tissue is muscles. There, they form macroand microcysts by proliferating via endopolygeny. The cysts are composed of partitions that contain bradyzoites. Cysts mostly develop in striated muscles, tongue, diaphragm, heart, and esophagus. Occasionally, cysts can be found in the brain. Cysts take approximately 75 days after infection to develop in intermediate hosts. The end hosts can only become infected by eating cysts containing bradyzoites (Dhaliwal and Juyal 2013; Sevgili 2015).

When the final host eats meat containing cysts, proteolytic enzymes in the digestive system break down the cysts and bradyzoites are released. They enter intestinal epithelial cells, where they pass the gametogony period. During this period, micro- and macrogametes are formed. Then the gametes are fertilized to form the zygote. At the end of this development process, oocysts with two sporocysts are produced. The wall of oocysts formed during the development of the Sarcocystis species is weak and thin. Therefore, the oocyst fragments while still in the host's intestine and sporocysts can be released. Thus, sporocysts can be excreted with feces. The prepatent period can be 7-14 days, and the patent period can vary from one week to several months (Fayer 2004; Dhaliwal and Juyal 2013; Sevgili 2015). 


\section{Clinical Signs in Humans}

Intestinal Sarcocystis infections in humans are usually asymptomatic. However, abdominal bloating, pain, nausea, vomiting, and diarrhea can occasionally occur in infected individuals. Humans sometimes serve as a random intermediate host for several Sarcocystis species, in which case muscular Sarcocystosis occurs. Mild muscular Sarcocystosis infections usually do not show any significant symptoms. However, in some patients, painful edema, erythema, fever, chronic myositis, myalgia, bronchospasm, lymphadenopathy, subcutaneous nodules, and eosinophilia can be seen (Sing et al. 2019).

\section{Clinical Signs in Animals}

Sarcocystis species, which are highly pathogenic for intermediate hosts, can sometimes cause death. The second period of merogony in the vascular endothelium of intermediate hosts is more pathogenic than cysts in the heart, nerve, and skeletal muscles. Severe acute infections often result in death. Cysts contain a toxin that is highly toxic to animals, such as rabbits, mice, and sparrows. In the case of cysts in intermediate hosts, myocarditis occurs in the heart muscles and myositis in other muscles. In addition, petechial bleeding occurs in the heart muscles and serosa during infections, and edema can occur in the lymph nodes. Macroscopic cysts in the muscles that are visible to the eye are considered to be less harmful. As time passes, macroscopic cysts become calcified. In cattle infections, symptoms such as anorexia, weakness, irregular fever, decreased milk yield, respiratory distress, and anemia are observed (Dhaliwal and Juyal 2013; Sevgili 2015). In chronic infections, weakening, edema under the chin, and exophthalmos may occur. Anorexia and weakening are seen in acute infections in sheep, while abortion can occur in severe infections (Sevgili 2015).

\section{Diagnosis}

Macrocysts between the muscles can easily be seen with the naked eye when examining the infected meat of intermediate host animals such as sheep, ducks, and rabbits. These visible cysts are 1-10 $\mathrm{mm}$ in size and white or ash in color. Cysts in other animals are microscopic, but can be seen in histological or pathological examination. When the disease is suspected in humans, biopsy material is examined from the blood vessels of various organs, including the kidney and heart. However, serological tests such as IFA and ELISA and molecular techniques such as PCR can also be used to diagnose infection in intermediate hosts (Sevgili 2015; Vasan and Tsuji 2009). In final host animals, stool examination is performed using the flotation method to determine the presence of sporulated oocysts or sporocysts (Fayer 2004).

\section{Treatment}

Since there are no symptoms to diagnose Sarcocystosis, the disease is usually hidden. Therefore, the use of medications for treatment is limited. Amprolium can be used at a dose of $100 \mathrm{mg} / \mathrm{kg}$ in the treatment of cats and dogs, which are end hosts. In addition, experimental studies have reported that halofuginone prevents severe infections (Sevgili 2015).

\section{Control and Prevention}

Humans can serve as both intermediate and final Sarcocystis hosts. To prevent infections in which humans are the final host, eating the raw or undercooked meat of animals such as sheep, goats, cattle, and pigs should be avoided. Meat suspected of being infected should be frozen or well-cooked to inactivate cysts. In meat cooked at $60-80^{\circ} \mathrm{C}$ for 15 minutes, bradyzoites lose their vitality. To prevent infection by Sarcocystis species in which humans are intermediate hosts, food and water contaminated with the feces of carnivorous or herbivorous animals should be avoided. Vegetables and fruit should be well washed. If water is not known to be uncontaminated, it should not be used for drinking. To protect intermediate host livestock from infection, contamination of shelters, feeders and water containers of these animals with the feces of the final hosts should be prevented. Animals such as cats and dogs should be prevented from entering such areas. To prevent infection in final host carnivores, these animals should not be given raw or undercooked meat or organs. Periodically, the stool of cats and dogs should be examined (Urquhart et al. 1996, Sevgili 2015, Ortega 2019, Sing et al. 2019).

\section{REFERENCES}

Allan JC et al., 1996. Field trial of the coproantigen-based diagnosis of Taenia solium taeniasis by enzyme linked immunosorbent assay. American Journal of Tropical Medicine and Hygiene 54:352-356.

Anantaphruti MT et al., 2007. Sympatric occurrence of Taenia solium, T. saginata, and T. asiatica. Emerging Infectious Diseases 13: 1413-1416.

Bern C et al., 1999. Magnitude of the disease burden from Neurocysticercosis in a developing country. Clinical Infectious Diseases 29: 1203-1209.

Bhatia BB et al., 2010. Food-borne parasitic zoonoses. In: Text Book of Veterinary Parasitology. 3rd Edition, pp: 601- 632.

Brandt JRA et al., 1992. A monoclonal antibody-based ELISA for the detection of circulating excretory secretory antigens in Taenia saginata cysticercosis. International Journal for Parasitology 22: 471-477.

Bruschi F and Gomez-Morales MA, 2017. Parasites. In: Foodborne Diseases, Dodd et al. (editors). Third Edition, Academic Press, London, UK, pp: 305-324.

Campbell WC, 1988. Trichinosis revisited: Another look at modes of transmission. Parasitology Today 4: 83-86.

Cardenas C et al., 1992. Taenia solium ocular cysticercosis: Findings in 30 cases. Annals of Ophthalmology 24: 25-28.

Dhaliwal BBS and Juyal PD, 2013. Parasitic Zoonoses. Springer, India. 
Dominique MLD et al., 20o8. Toxoplasma gondii. In: Khan NA (editor). Emerging Protozoan Pathogens. Taylor \& Francis.

Dubey JP et al., 1986. Distribution of Toxoplasma gondii cysts in commercial cuts of pork. Journal of the American Veterinary Medical Association 188: 1035 .

Dubey JP et al., 1990. Effect of high temperature on infectivity of Toxoplasma gondii tissue cysts in pork. The Journal of Parasitology 76: 201-204.

Dubey JP and Beattie CP, 1988. Toxoplasma and toxoplasmosis in animals including man. CRC Press, Florida, USA.

Dubey JP and Thayer DW, 1994. Killing of different strains of Toxoplasma gondii tissue cysts by irradiation under defined conditions. The Journal of Parasitology 80: 764-767.

Dubey JP, 1986. A review of Toxoplasmosis in cattle. Veterinary Parasitology 22: 177-202.

Dubey JP, 1998. Toxoplasmosis, sarcocystiosis, isosporosis and cyclosporosis. In: Zoonoses: Biology, Clinical Practice, and Public Health Control. SR Palmer, EJL Soulsby, DIH Simpson (editors). Oxford University Press, Oxford, UK, pp: 579-597.

Dubey JP, 2015. Foodborne and waterborne zoonotic sarcocystosis. Food and Waterborne Parasitology 1: 211.

Dumanlı A and Aktaş M, 2015. Toxoplasmatidae (Toxoplasma, Neospora). In: Dumanlı N, Karaer KZ (editors) Veterinary Protozoology. Medisan Publication Series: 8o, Second Edition, Ankara, Turkey.

Dupouy-Camet J, 20oo. Trichinellosis: A Worldwide Zoonosis. Veterinary Parasitology 93: 191-200.

Elias FM et al., 2005. Oral cysticercosis: Case report and review of the literature. The Revista do Instituto de Medicina Tropical de São Paulo 47: 95-98.

Erol İ, 2007. Food Hygiene and Microbiology. Ankara University, Faculty of Veterinary, Department of Food Hygiene and Technology, Ankara.

Fayer R, 1994. Foodborne and waterborne zoonotic protozoa. In: Foodbome Diseases Handbook. Volume 2: Diseases caused by viruses, parasites, and fungi. $\mathrm{YH}$ Hui, JR Gorham, KD, Murrell, DO Oliver, (editors), Marcel Dekker, New York, USA, pp: 331-362.

Fayer R, 2004. Sarcocystis spp. In human infections. Clinical Microbiology Reviews 17: 894-902.

Flisser A et al., 2005. Evaluation of a self-detection tool for tapeworm carriers for use in public health. American Journal of Tropical Medicine and Hygiene 72: 510-512.

Galán-Puchades MT and Fuentes MV, 2013. Lights and shadows of the Taenia asiatica life cycle and pathogenicity. Tropical Parasitology 3: 114-119.

Galán-Puchades MT and Fuentes MV, 2014. Taenia asiatica: Left out by globalisation? Trends in Parasitology 30: 54-55.

Gajadhar AA et al., 20o6. Overview of food- and waterborne zoonotic parasites at the farm level. Revue Scientifique et Technique 25: 595-6o6.
Gajadhar AA et al., 2009. Trichinella diagnostics and control: Mandatory and best practices for ensuring food safety. Veterinary Parasitology 159: 197-205.

Gamble HR et al., 1996. Methods for the detection of Trichinellosis in horses. Journal of Food Protection 59: 420-425.

Gamble HR, 1998. Sensitivity of artificial digestion and enzyme immunoassay methods of inspection for Trichinae in pigs. Journal of Food Protection 61: 339343.

Garcia HH et al., 2003. Taenia solium Cysticercosis. The Lancet 362: 547-556.

Gottstein B et al., 1991. Diagnostic identification of Taenia saginata with the polymerase chain reaction. Transactions of the Royal Society of Tropical Medicine and Hygiene 85: 248-249.

Gupta RK et al., 2018. Meat borne parasitic zoonoses: Epidemiology, diagnosis, prevention, current issues and approaches. International Journal of Veterinary Sciences and Animal Husbandry 3: 86-88.

Harrison LJS et al., 1989. Specific detection of circulating surface/secreted glycoproteins of viable cysticerci in Taenia saginata Cysticercosis. Parasite Immunology 11: 351-370.

Hohlfeld P et al., 1989. Fetal toxoplasmosis: Outcome of pregnancy and infant follow-up after in utero treatment. The Journal of Pediatrics 115: 765-769.

Joynson DH and Wreghitt TG, 2005. Toxoplasmosis: A comprehensive clinical guide. Cambridge University Press, Cambridge, UK.

Juyal PD et al., 20o8. Epidemiology and control strategies against Cysticercosis (due to Taenia solium) with special reference to swine and human in Asia. Journal of Veterinary and Animal Sciences 1: 1-10.

Li $\mathrm{T}$ et al., 2007. Taeniasis/cysticercosis in China. The Southeast Asian Journal of Tropical Medicine and Public Health 38: 131-139.

Liu D, 2019. Taenia. In: Handbook of Foodborne Diseases. Liu D. (editor), CRC Press Taylor \& Francis Group, New York, USA, pp: 715-720.

Macpherson $\mathrm{CN}$ et al., 20oo. Parasitic food-borne and water-borne zoonoses. Revue Scientifique et Technique 19: 240-258.

McCabe RE, 2001. Antitoxoplasma chemotherapy. In: Toxoplasmosis. A comprehensive clinical guide. DHM Joynson and TG Wreghitt (editors), Cambridge University Press, Cambridge, UK, pp: 319-359.

Murrell KD, 2005. Epidemiology of Taeniosis and Cysticercosis. In: Murrell KD (editor) WHO/FAO/OIE Guidelines for the Surveillance, Prevention and Control of Taeniosis. OIE, Paris, pp: 27-32.

Ortega YR, 2019. Protozoan parasites. In: Food Microbiology: Fundamentals and Frontiers. Doyle MP, Diez-Gonzalez F and Hill C (editors), 5th Edition. ASM Press, Washington, DC, USA, pp: 667695.

Parija SC, 2009. A textbook of Medical Parasitology. Third Edition. All India Publishers and Distributors, New Dehli, India. 
Pozio E, 1998. Trichinellosis in the European Union: Epidemiology, ecology and economic impact. Parasitology Today 14: 35-38.

Pozio E and Bruschi F, 2019. Trichinella. In: Handbook of Foodborne Diseases. Liu D (editor), CRC Press, Taylor \& Francis Group, New York, USA, pp: 887-897.

Prasad KJ, 2010. Emerging and re-emerging parasitic diseases. Journal of International Medical Sciences Academy 23(1): 45-50.

Rommel $\mathrm{M}$ et al., 200o. Veterinarmedizinische Parasitologie. Parey Buchverlag, Berlin.

Sayg1 G, 1999. Taeniosis ve Etkenleri. Cumhuriyet Üniversitesi Rektörlük Basımevi, Sivas, pp. 164.

Schantz PM et al., 1998. Immigrants, imaging and immunoblots: The emergence of Neurocysticercosis as a significant health problem. In: Emerging Infections 2. WM Scheid, D Armstrong, JB Hughes (editors). ASM Press, Washington DC, USA, pp: 213242.

Schantz PM et al., 1993. Potential eradicability of Taeniasis and Cysticercosis. Bulletin Pan American Health Organization 27: 397-403.

Sevgili M, 2015. Sarcocystiade (Sarcocystis, Frenkelia). Dumanlı N, Karaer KZ (editors), Veterinary Protozoology. Medisan Publication Series: 8o, Second Edition, Ankara, Turkey.

Shakespeare M, 2009. Zoonose. Second Edition; Pharmaceutical Press, London, UK.

Sing BB et al., 2019. Sarcocystis. In: Handbook of Foodborne Diseases. Liu D (editor), CRC Press Taylor \& Francis Group, New York, USA; pp: 631-640.

Slifko TR et al., 200o. Emerging parasite zoonoses associated with water and food. The International Journal for Parasitology 30: 1379-1393.
Souza FS and Damatta RA, 2019. Toxoplasma. In: Handbook of Foodborne Diseases. Liu D (editor), CRC Press Taylor \& Francis Group, New York, USA; pp: 641-653.

Şenlik B, 2011. Teşhis Yöntemleri. In: Veteriner Helmintoloji. Editör; Tınar, R, Dora Basın-YayınDağıtım, Bursa/Turkey; pp: 427-482.

Şenlik B, 2017. Koyunlarda Sürü Sağlı̆̆ı Açısından Önemli Paraziter Hastalıklar. Türkiye Klinikleri, Veterinary Sciences, Special Topics Journal 3: 89-100.

Tsang VCW and Wilson M, 1995. Taenia solium Cysticercosis: An under-recognized but serious public health problem. Parasitology Today 11: 124-126.

Urquhart GM et al., 1996. Veterinary Parasitology. Blackwell Science, The Faculty of Veterinary Medicine. The University of Glasgow Scotland, UK; pp: $220-387$.

Ünüvar S, 2018. Microbial Foodborne Diseases. In: Foodborne Diseases. Holban AM, Grumezescu, (editors), Academic Press, an imprint of Elsevier, London, UK; pp: 1-28.

Vasan S and Tsuji M, 2009. Toxoplasmosis. In: Satoskar AR, Simon GL, Hotez PJ, Tsuji M (editors), Medical Parasitology. Landes Bioscience, USA.

Yanagida T et al., 2012. Taeniasis and Cysticercosis due to Taenia solium in Japan. Parasites Vectors 5: 18.

Zarlenga DS and Gamble, 2019. Helminths in meat. In: Food Microbiology: Fundamentals and Frontiers. $5^{\text {th }}$ Edition; Doyle MP, Diez-Gonzalez F and Hill C (editors), ASM Press, Washington, DC, USA; pp: 645665 .

Zhou P et al., 20o8. Food-borne parasitic zoonoses in China: Perspective for control. Trends in Parasitology 24: 190-196. 